Published in final edited form as:

Transplantation. 2018 August ; 102(8): e368-e369. doi:10.1097/TP.0000000000002271.

\title{
Response to commentary
}

\author{
Peter S. Heeger, MD \\ Icahn School of Medicine at Mount Sinai
}

In response to the editorial comments by Johnson and Kaplan ${ }^{1}$ regarding the results of our recently published CTOT17 analysis ${ }^{2}$, we offer the following reply.

We agree with the comments that "a biomarker needs to be a strong predictor of the clinical endpoint ....and also that a change in the biomarker predicts the change in the outcome; to achieve this, the biomarker must lie on the causal pathway of disease." However, Johnson and Kaplan incorrectly state that we tested a change in eGFR as a surrogate biomarker for transplant outcomes.

Our goals in the project were two-fold. First, we tested whether candidate biomarkers including donor-reactive ELISPOT assays and urinary chemokine assays performed within the initial 2 years posttransplant correlated with 5-year outcomes. Our analysis showed no relationships, despite strong relationships between the candidate biomarkers and the 2-year outcomes. ${ }^{3,4}$ These negative results serve as an important caution for design of future biomarker studies.

Secondly, we addressed the possibility that changes in eGFR over time posttransplant could be used, not as a biomarker but rather, as a surrogate endpoint for the "hard" endpoint of graft failure. A surrogate endpoint differs from a biomarker, and as such, the implications differ. As defined by Flemings and Powers 5 (ref 6 in the Johnson/Kaplan editorial): “A surrogate endpoint is an outcome measure 'used as a substitute for a clinically meaningful endpoint...changes induced by a therapy on a surrogate endpoint are expected to reflect changes in a clinically meaningful endpoint.",

In our case, the clinically meaningful endpoint was graft loss and the candidate surrogate endpoint was a change in eGFR of $\geq 30 \%$ between 6-months and 2-years posttransplant. Graft loss was not defined by an "arbitrary GFR" but rather was defined by each participating clinical site as allograft function that was so poor as to require dialysis, relisting or retransplantation (the definition accepted by UNOS).

We reasoned that additional evidence supporting changes in posttransplant eGFR within the first 2-years as a surrogate endpoint for graft loss at 5-years would have clinically relevant implications. As one example, future interventional clinical trials in kidney transplant recipients could be designed such that they employ the change in eGFR within 2 years as a 
surrogate endpoint rather than require a 5-year (or greater) wait to test for the hard endpoint of graft loss.

While we acknowledge the weakness that relatively few patients in CTOT17 reached the 5year outcome of graft loss, we contend that in conjunction with a) large datasets in subjects with native kidney disease ${ }^{6}$ and b) a separate analysis of kidney transplant outcomes from Australia $^{7}$, our data add to the accumulating evidence that a $30-40 \%$ change in eGFR between 6-months and 2-years posttransplant could be a useful surrogate endpoint for graft loss.

\section{References}

1. Johnson C, Kaplan B. Biomarkers and Kidney Transplant: Time for a New Paradigm? Transplantation. 2018; 102(4):552-553. [PubMed: 29319617]

2. Faddoul G, Nadkarni GN, Bridges ND, et al. Analysis of Biomarkers Within the Initial 2 Years Posttransplant and 5-Year Kidney Transplant Outcomes: Results From Clinical Trials in Organ Transplantation-17. Transplantation. 2018; 102(4):673-680. [PubMed: 29189482]

3. Hricik DE, Nickerson P, Formica RN, et al. Multicenter validation of urinary CXCL9 as a riskstratifying biomarker for kidney transplant injury. Am J Transplant. 2013; 13(10):2634-2644. [PubMed: 23968332]

4. Hricik DE, Augustine J, Nickerson P, et al. Interferon Gamma ELISPOT Testing as a RiskStratifying Biomarker for Kidney Transplant Injury: Results From the CTOT-01 Multicenter Study. Am J Transplant. 2015; 15(12):3166-3173. [PubMed: 26226830]

5. Fleming TR, Powers JH. Biomarkers and surrogate endpoints in clinical trials. Stat Med. 2012; 31(25):2973-2984. [PubMed: 22711298]

6. Coresh J, Turin TC, Matsushita K, et al. Decline in estimated glomerular filtration rate and subsequent risk of end-stage renal disease and mortality. JAMA. 2014; 311(24):2518-2531. [PubMed: 24892770]

7. Clayton PA, Lim WH, Wong G, Chadban SJ. Relationship between eGFR Decline and Hard Outcomes after Kidney Transplants. J Am Soc Nephrol. 2016; 27(11):3440-3446. [PubMed: 27059513] 